Article

\title{
One Type of Symmetric Matrix with Harmonic Pell Entries, Its Inversion, Permanents and Some Norms
}

\author{
Seda Yamaç Akbiyik 1,*,+(D), Mücahit Akbiyik ${ }^{2,+}$ (D) and Fatih Yilmaz ${ }^{3,+}$ (D) \\ 1 Department of Computer Engineering, Istanbul Gelisim University, 34310 Istanbul, Turkey \\ 2 Department of Mathematics, Beykent University, 34520 Istanbul, Turkey; mucahitakbiyik@beykent.edu.tr \\ 3 Department of Mathematics, Ankara Hacı Bayram Veli University, 06900 Ankara, Turkey; \\ fatih.yilmaz@hbv.edu.tr \\ * Correspondence: syamac@gelisim.edu.tr \\ + These authors contributed equally to this work.
}

check for updates

Citation: Yamaç Akbiyik, S.; Akbiyik, M.; Yilmaz, F. One Type of Symmetric Matrix with Harmonic Pell Entries, Its Inversion, Permanents and Some Norms. Mathematics 2021, 9, 539. https://doi.org/10.3390/math9050539

Academic Editor: Juan Ramón Torregrosa Sánchez

Received: 4 February 2021

Accepted: 2 March 2021

Published: 4 March 2021

Publisher's Note: MDPI stays neutral with regard to jurisdictional claims in published maps and institutional affiliations.

Copyright: (C) 2021 by the authors Licensee MDPI, Basel, Switzerland. This article is an open access article distributed under the terms and conditions of the Creative Commons Attribution (CC BY) license (https:/ / creativecommons.org/licenses/by/ $4.0 /)$.

\begin{abstract}
The Pell numbers, named after the English diplomat and mathematician John Pell, are studied by many authors. At this work, by inspiring the definition harmonic numbers, we define harmonic Pell numbers. Moreover, we construct one type of symmetric matrix family whose elements are harmonic Pell numbers and its Hadamard exponential matrix. We investigate some linear algebraic properties and obtain inequalities by using matrix norms. Furthermore, some summation identities for harmonic Pell numbers are obtained. Finally, we give a MATLAB-R2016a code which writes the matrix with harmonic Pell entries and calculates some norms and bounds for the Hadamard exponential matrix.
\end{abstract}

Keywords: harmonic pell number; spectral norm; hadamard inverse; permanent; determinant

\section{Introduction}

The Pell numbers [1] which are defined by the recurrence relation, for $n \geq 0$ :

$$
P_{n+2}=2 P_{n+1}+P_{n}
$$

with $P_{0}=0, P_{1}=1$, provide the mathematical community with their magnificent beauty, ubiquity and applicability, offering great opportunities to experiment, explore and solve problems, and their delightful applications appear widely in the literature. In [2], the author considers a $k$-circulant matrix whose first row is $\left(P_{1}, P_{2}, \ldots, P_{n}\right)$, where $P_{n}$ is the $n^{\text {th }}$ Pell number, and obtain the formulae for the eigenvalues of such matrix. In [3], the authors introduce a new type of matrix called circulant-like matrix whose entries are written as functions of Horadam, Fibonacci, Jacobsthal and Pell numbers. Furthermore, they investigate some algebraic properties. It is known that the $n$th harmonic number $H_{n}$, ref. [4] has the usual definition

$$
H_{n}=\sum_{k=1}^{n} \frac{1}{k}=\sum_{j=1}^{\infty} \frac{n}{j(j+n)}=\int_{0}^{1} \frac{1-x^{n}}{1-x} d x \quad\left(H_{0}=0\right)
$$

for $n \in \mathbb{N}$, where $\mathbb{N}$ denotes the set of natural numbers without zero. The harmonic numbers and their generalizations have been studied for many years and cover a wide range of fields, such as computer science, number theory, physics, and matrix theory. In [5], the authors define $n$ by $n$ matrices associated with harmonic numbers, they obtain some properties for their norms. In [6], some spectral bounds for the harmonic matrix are obtained. Furthermore, the circulant and $r$-circulant matrices whose entries are harmonic and hyperharmonic Fibonacci numbers are studied [7].

Matrix theory and linear algebra are fundamental tools in many mathematical disciplines. One can find a lot of basic topics for a variety of these areas in [8-10], in details. In 
the literature, it is quite common to construct different matrix types from past to present and to examine the properties of those matrices such as norms and permanents, etc. In [11], Hadamard exponential Hankel matrix is defined and its eigenvalues and some norms are examined. In [12], the spectral norms of the matrices connected integer numbers sequences are stated. In [13], the authors investigate the spectral norms of Toeplitz matrices with Fibonacci and Lucas numbers. In [14], the authors study on permanents of the matrices. Furthermore, Brualdi et al. define a new method to compute permanents for some type of matrices, in [15]. It is known that there are a lot of relationships between determinants or permanents of matrices and well-known number sequences. In [16], the authors study on Fibonacci and related sequences in periodic tridiagonal matrices. In [17], the authors investigate relationships between permanents of one type of Hessenberg matrix and the Pell and Perrin numbers. Petroudi et al. define a special symmetric matrix form and its Hadamard exponential matrix. Furthermore, they give inverses and some norms for the matrices, in [18].

In this paper, by inspiring the definition of harmonic numbers, we define harmonic Pell numbers. Firstly, we construct a new type of symmetric matrix whose entries are the harmonic Pell numbers and its Hadamard exponential matrix. Secondly, we give the determinants of these matrices. Furthermore, we find the leading principle minors and inverses of them. Then, some bounds for the norm of these matrices are obtained. Moreover, we give an illustrative example for all results. Finally, we present a MATLAB-R2016a code which writes the matrix with harmonic Pell entries and calculates some norms and bounds for the Hadamard exponential matrix.

\section{Preliminaries}

The norm of a matrix is a non-negative real number which is a measure of the magnitude of the matrix. There are several different ways of defining a matrix norm but they all share the same certain properties. Let $A=\left(a_{i j}\right)$ be an $n$ by $n$ matrix, then the maximum column norm is

$$
\|A\|_{c_{1}}=\max _{j} \sqrt{\sum_{i}\left|a_{i j}\right|^{2}}
$$

and the maximum row norm is

$$
\|A\|_{r_{1}}=\max _{i} \sqrt{\sum_{j}\left|a_{i j}\right|^{2}} .
$$

The $\ell_{p}$ norm of $A$ is defined by

$$
\|A\|_{p}=\left(\sum_{i=1}^{n} \sum_{j=1}^{n}\left|a_{i j}\right|^{p}\right)^{\frac{1}{p}} .
$$

For $p=2$, the $\ell_{p}$ norm is defined as Euclidean (Frobenius) norm and is denoted by $\|A\|_{\mathbb{E}}$. Let $A^{H}$ be the conjugate transpose of matrix $A$ and $\lambda_{i}$ be the eigenvalue of matrix $A A^{H}$, then the spectral norm of the matrix $A$ is

$$
\|A\|_{2}=\sqrt{\max _{1 \leq i \leq n} \lambda_{i}}
$$

If the matrix $A$ equals to the Hadamard product of the matrices $B$ and $C$, (i.e., $A=B \circ C=$ $\left.\left(b_{i j} c_{i j}\right)\right)$, then the following relation is satisfied

$$
\|A\|_{2} \leq\|B\|_{r_{1}}\|C\|_{c_{1}} .
$$

The Frobenius and spectral norm of the matrix $A$ satisfy the following inequality:

$$
\frac{1}{\sqrt{n}}\|A\|_{\mathbb{E}} \leq\|A\|_{2} \leq\|A\|_{\mathbb{E}}
$$


Additionally, the Hadamard exponential matrix of the matrix $A=\left(a_{i j}\right)_{m \times n}$ is defined by $e^{\circ A}=\left(e^{a_{i j}}\right)[10]$. Assume that $M$ is a square matrix as below:

$$
M=\left[\begin{array}{cc}
A & b \\
b^{T} & c
\end{array}\right]
$$

where $A$ is an $n$ by $n$ non-singular matrix and $b$ is an $n \times 1$ matrix, also $c$ is a real number. Then, the inverse of $M$ is

$$
N=\left[\begin{array}{cc}
A^{-1}+\frac{1}{l} A^{-1} b b^{T} A^{-1} & -\frac{1}{l} A^{-1} b \\
-\frac{1}{l} b^{T} A^{-1} & c
\end{array}\right]
$$

where $l=c-b^{T} A^{-1} b$ [18]. Moreover, the permanent of an $n$ by $n$ matrix $A$ is defined as

$$
\operatorname{per} A=\sum_{\sigma \in S_{n}} \prod_{i=1}^{n} a_{i, \sigma(i)}
$$

the sum here extends over all elements $\sigma$ of the symmetric group $S_{n}$ over all permutations of the numbers $1,2, \ldots, n,[14]$.

\section{Harmonic Pell Numbers and Some Symmetric Matrices}

Let us define the $n$th harmonic Pell number by

$$
\mathbb{P}_{n}=\sum_{k=1}^{n} \frac{1}{P_{k}}
$$

where $P_{k}$ is the $k$ th Pell number. Additionally, let us construct two symmetric $n$ by $n$ matrix denoted by $\mathfrak{P}=\left[\mathbb{P}_{k_{i, j}}\right]_{i, j=1}^{n}$ and the Hadamard exponential matrix denoted by $e^{\circ \mathfrak{P}}=\left[e^{\mathbb{P}_{k_{i, j}}}\right]_{i, j=1}^{n}$ of the matrix $\mathfrak{P}$, respectively, as below:

$$
\mathfrak{P}=\left[\begin{array}{ccccc}
\mathbb{P}_{1} & \mathbb{P}_{1} & \mathbb{P}_{1} & \cdots & \mathbb{P}_{1} \\
\mathbb{P}_{1} & \mathbb{P}_{2} & \mathbb{P}_{2} & \cdots & \mathbb{P}_{2} \\
\mathbb{P}_{1} & \mathbb{P}_{2} & \mathbb{P}_{3} & \cdots & \mathbb{P}_{3} \\
\vdots & \vdots & \vdots & \vdots & \vdots \\
\mathbb{P}_{1} & \mathbb{P}_{2} & \mathbb{P}_{3} & \cdots & \mathbb{P}_{n}
\end{array}\right]=\left[\begin{array}{ccccc}
1 & 1 & 1 & \cdots & 1 \\
1 & \frac{3}{2} & \frac{3}{2} & \cdots & \frac{3}{2} \\
1 & \frac{3}{2} & \frac{17}{10} & \cdots & \frac{17}{10} \\
\vdots & \vdots & \vdots & \vdots & \vdots \\
1 & \frac{3}{2} & \frac{17}{10} & \cdots & \sum_{k=1}^{n} \frac{1}{P_{k}}
\end{array}\right]
$$

and

$$
e^{\circ \mathfrak{P}}=\left[\begin{array}{ccccc}
e^{\mathbb{P}_{1}} & e^{\mathbb{P}_{1}} & e^{\mathbb{P}_{1}} & \cdots & e^{\mathbb{P}_{1}} \\
e^{\mathbb{P}_{1}} & e^{\mathbb{P}_{2}} & e^{\mathbb{P}_{2}} & \cdots & e^{\mathbb{P}_{2}} \\
e^{\mathbb{P}_{1}} & e^{\mathbb{P}_{2}} & e^{\mathbb{P}_{3}} & \cdots & e^{\mathbb{P}_{3}} \\
\vdots & \vdots & \vdots & \vdots & \vdots \\
e^{\mathbb{P}_{1}} & e^{\mathbb{P}_{2}} & e^{\mathbb{P}_{3}} & \cdots & e^{\mathbb{P}_{n}}
\end{array}\right]
$$

where $k_{i, j}=\min \{i, j\}$ and $\mathbb{P}_{k}$ is the $k$ th harmonic Pell number. We give some values of the harmonic and harmonic Pell numbers in Table 1.

Table 1. Some harmonic and harmonic Pell numbers.

\begin{tabular}{ccccccccccc}
\hline $\boldsymbol{n}$ & $\mathbf{1}$ & $\mathbf{2}$ & $\mathbf{3}$ & $\mathbf{4}$ & $\mathbf{5}$ & $\mathbf{6}$ & $\mathbf{7}$ & $\mathbf{8}$ & $\mathbf{9}$ & $\mathbf{1 0}$ \\
\hline$H_{n}$ & 1 & $\frac{3}{2}$ & $\frac{11}{6}$ & $\frac{25}{12}$ & $\frac{137}{60}$ & $\frac{49}{20}$ & $\frac{363}{140}$ & $\frac{761}{280}$ & $\frac{7129}{2520}$ & $\frac{83,711}{27,720}$ \\
\hline $\mathbb{P}_{n}$ & 1 & $\frac{3}{2}$ & $\frac{17}{10}$ & $\frac{107}{60}$ & $\frac{449}{247}$ & $\frac{251}{137}$ & $\frac{261}{142}$ & $\frac{173}{94}$ & $\frac{151}{82}$ & $\frac{501}{272}$ \\
\hline
\end{tabular}


Theorem 1. Let $\mathfrak{P}$ be an $n$ by $n$ matrix given in (10). Then,

$$
\operatorname{det}(\mathfrak{P})=\frac{1}{P_{1} P_{2} \ldots P_{n}}
$$

where $P_{n}$ is the $n$th Pell number.

Proof. By applying the elementary row operations on the matrix (10), in other words by multiplying each row with -1 and adding it to the consecutive row, we obtain:

$$
\operatorname{det}(\mathfrak{P})=\operatorname{det}\left[\begin{array}{ccccc}
\mathbb{P}_{1} & \mathbb{P}_{1} & \mathbb{P}_{1} & \cdots & \mathbb{P}_{1} \\
0 & \mathbb{P}_{2}-\mathbb{P}_{1} & \mathbb{P}_{2}-\mathbb{P}_{1} & \cdots & \mathbb{P}_{2}-\mathbb{P}_{1} \\
0 & 0 & \mathbb{P}_{3}-\mathbb{P}_{2} & \cdots & \mathbb{P}_{3}-\mathbb{P}_{2} \\
\vdots & \vdots & \vdots & \vdots & \vdots \\
0 & 0 & 0 & \cdots & \mathbb{P}_{n}-\mathbb{P}_{n-1}
\end{array}\right]
$$

So, we get the following equation:

$$
\operatorname{det}(\mathfrak{P})=\mathbb{P}_{1} \prod_{i=2}^{n}\left(\mathbb{P}_{i+1}-\mathbb{P}_{i}\right)=\prod_{i=1}^{n} \frac{1}{\mathbb{P}_{i}}=\frac{1}{P_{1} P_{2} \ldots P_{n}}
$$

Theorem 2. The leading principal minor $\Delta_{n}$ of the matrix $\mathfrak{P}$ which is given in (10) satisfies the followings:

$$
\begin{array}{ll}
\text { i. } & \Delta_{n}=\frac{1}{P_{n}} \Delta_{n-1}, \\
\text { ii. } & \Delta_{1} \Delta_{2} \Delta_{3} \cdots \Delta_{n}=\frac{1}{P_{1}^{n} P_{2}^{n-1} \ldots P_{n-1}^{2} P_{n}} .
\end{array}
$$

Proof. By exploiting Theorem 1 and the equations given below:

$$
\Delta_{1}=1, \Delta_{2}=\frac{1}{2}, \Delta_{3}=\frac{1}{10}, \cdots, \Delta_{n}=\frac{1}{P_{1} P_{2} \ldots P_{n}},
$$

the proof is clear.

Corollary 1. Let $\mathfrak{P}$ be a matrix which is given in (10), then $\mathfrak{P}$ is a positively defined matrix. All eigenvalues of $\mathfrak{P}$ are positive.

Theorem 3. Let $\mathfrak{P}$ be a matrix as in the matrix (10), then the inverse of $\mathfrak{P}$ is calculated as

$$
\mathfrak{P}^{-1}=\left[\begin{array}{cccccccc}
P_{1}+P_{2} & -P_{2} & 0 & 0 & 0 & \cdots & 0 & 0 \\
-P_{2} & P_{2}+P_{3} & -P_{3} & 0 & 0 & \cdots & 0 & 0 \\
0 & -P_{3} & P_{3}+P_{4} & -P_{4} & 0 & 0 & \cdots & 0 \\
0 & 0 & -P_{4} & P_{4}+P_{5} & -P_{5} & 0 & \cdots & 0 \\
\vdots & \vdots & \vdots & \vdots & \vdots & \vdots & \vdots & \vdots \\
0 & 0 & 0 & \cdots & 0 & -P_{n-1} & P_{n-1}+P_{n} & -P_{n} \\
0 & 0 & 0 & \cdots & 0 & 0 & -P_{n} & P_{n}
\end{array}\right] .
$$

Proof. By Theorem 1, it is known that $\mathfrak{P}$ is invertible. Note that the determinant of the inverse of $\mathfrak{P}$ is

$$
\operatorname{det}\left(\mathfrak{P}^{-1}\right)=P_{1} P_{2} \ldots P_{n} .
$$

The inverse of the matrix $\mathfrak{P}$ can be calculated by principle mathematical induction (PMI), on $n$. It verifies for $n=2$, i.e., if

$$
\mathfrak{P}=\left[\begin{array}{ll}
1 & 1 \\
1 & \frac{3}{2}
\end{array}\right]
$$


then we find

$$
\mathfrak{P}^{-1}=\left[\begin{array}{cc}
3 & -2 \\
-2 & 2
\end{array}\right]
$$

Suppose that the hypothesis is provided for $n$, that is, $A=\mathfrak{P}=\left[\mathbb{P}_{k_{i, j}}\right]_{n \times n}$, and there exists $A^{-1}=\left[\mathbb{P}_{k_{i, j}}\right]_{n \times n}^{-1}$. So, we have $b=\left(\mathbb{P}_{1}, \mathbb{P}_{2}, \cdots, \mathbb{P}_{n}\right)^{T}, \quad b^{T}=\left(\mathbb{P}_{1}, \mathbb{P}_{2}, \cdots, \mathbb{P}_{n}\right)$. By taking $c=\mathbb{P}_{n+1}$ and by the help of the Equation (8), the proof is completed for $n+1$.

Let us define a second order recurrence relation, for $i \geq 2$, as below:

$$
v^{[i]}=v^{[i-1]}\left(P_{i+3}+P_{i+4}\right)+v^{[i-2]} P_{i+3}^{2}
$$

where $v^{[1]}=v^{[0]}\left(P_{4}+P_{5}\right)+A P_{4}^{2}, v^{[0]}=A\left(P_{3}+P_{4}\right)+P_{3}^{2}\left(P_{1}+P_{2}\right)$ and $A=25$. The first few values of the sequence can be given as following:

$$
\begin{aligned}
& v^{[1]}=v^{[0]}\left(P_{4}+P_{5}\right)+A P_{4}^{2} \\
& v^{[2]}=v^{[1]}\left(P_{5}+P_{6}\right)+v^{[0]} P_{5}^{2} \\
& v^{[3]}=v^{[2]}\left(P_{6}+P_{7}\right)+v^{[1]} P_{6}^{2}
\end{aligned}
$$

where $i=2,3,4, \ldots$. Here, we construct a new recurrence relation whose permanents are related to inverse of the matrix $\mathfrak{P}^{-1}$.

Theorem 4. For $n \geq 5$, the permanents of the matrix $\mathfrak{P}^{-1}$ are:

$$
\operatorname{per}\left(\mathfrak{P}^{-1}\right)=P_{n}\left(v^{[n-4]}+v^{[n-5]} P_{n}\right) .
$$

For, $2 \leq n<5$ the permanent of $\mathfrak{P}^{-1}$ is calculated as follows:

$$
\begin{aligned}
& n=2, \quad \operatorname{per}\left(\mathfrak{P}^{-1}\right)=10, \\
& n=3, \quad \operatorname{per}\left(\mathfrak{P}^{-1}\right)=200, \\
& n=4, \quad \operatorname{per}\left(\mathfrak{P}^{-1}\right)=18100 .
\end{aligned}
$$

Proof. Let us consider $A=25$ and $v^{[0]}=A\left(P_{3}+P_{4}\right)+P_{3}^{2}\left(P_{1}+P_{2}\right)$, then by using a new method which is called as contraction method, we get

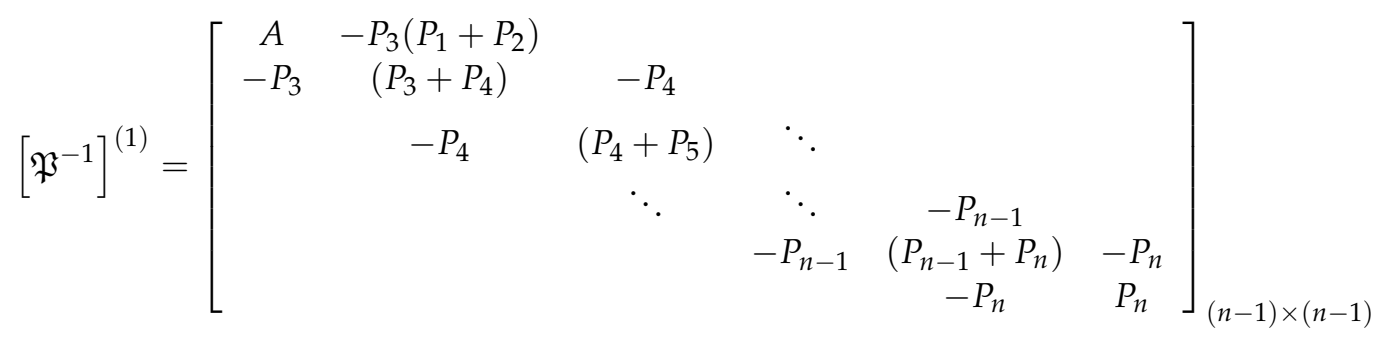

and going on with this method, we obtain

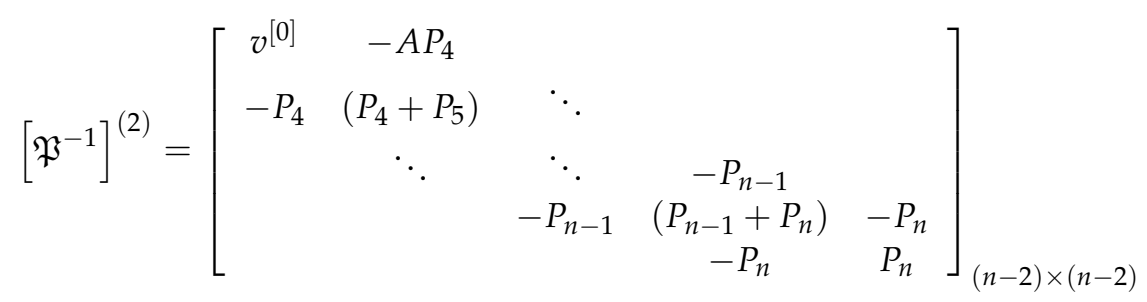


and we can mention these steps with a general statement, for $n-2>r \geq 3$, as below:

$$
\left[\mathfrak{P}^{-1}\right]^{(r)}=\left[\begin{array}{ccccc}
v^{[r-2]} & -v^{[r-3]} P_{r+2} & & & \\
-P_{r+2} & \left(P_{r+2}+P_{r+3}\right) & -P_{r+3} & & \\
& \ddots & \ddots & \ddots & \\
& & -P_{n-1} & \left(P_{n-1}+P_{n}\right) & -P_{n} \\
& & & -P_{n} & P_{n}
\end{array}\right]_{(n-r) \times(n-r)}
$$

Consequently, we get

$$
\left[\mathfrak{P}^{-1}\right]^{(n-2)}=\left[\begin{array}{cc}
v^{[n-4]} & -v^{[n-5]} P_{n} \\
-P_{n} & P_{n}
\end{array}\right]
$$

So, the permanent of $\mathfrak{P}^{-1}$ is

$$
\operatorname{per}\left(\mathfrak{P}^{-1}\right)=P_{n}\left(v^{[n-4]}+v^{[n-5]} P_{n}\right) .
$$

Similarly, for the special cases $2 \leq n<5$, the permanent of $\mathfrak{P}^{-1}$ can be easily found by using the same method.

Let us define the Hadamard inverse of a matrix $\mathfrak{P}$, as follows:

$$
\mathfrak{P}^{\circ}(-1)=\left[\begin{array}{ccccc}
\frac{1}{\mathbb{P}_{1}} & \frac{1}{\mathbb{P}_{1}} & \frac{1}{\mathbb{P}_{1}} & \cdots & \frac{1}{\mathbb{P}_{1}} \\
\frac{1}{\mathbb{P}_{1}} & \frac{1}{\mathbb{P}_{2}} & \frac{1}{\mathbb{P}_{2}} & \cdots & \frac{1}{\mathbb{P}_{2}} \\
\frac{1}{\mathbb{P}_{1}} & \frac{1}{\mathbb{P}_{2}} & \frac{1}{\mathbb{P}_{3}} & \cdots & \frac{1}{\mathbb{P}_{3}} \\
\vdots & \vdots & \vdots & \vdots & \vdots \\
\frac{1}{\mathbb{P}_{1}} & \frac{1}{\mathbb{P}_{2}} & \frac{1}{\mathbb{P}_{3}} & \cdots & \frac{1}{\mathbb{P}_{n}}
\end{array}\right]
$$

where $\mathbb{P}_{n}$ is the $n$th harmonic Pell number.

Theorem 5. Assume that $\mathfrak{P}$ is a matrix which is given in (10). Then,

$$
\operatorname{det}\left(\mathfrak{P}^{\circ(-1)}\right)=\frac{(-1)^{n-1}}{\prod_{i=1}^{n-1} P_{i} \mathbb{P}_{i}^{2} \mathbb{P}_{n}}
$$

Proof. By multiplying each row with -1 and adding it to the consecutive row, we obtain:

$$
\operatorname{det}\left(\mathfrak{P}^{\circ}(-1)\right)=\operatorname{det}\left[\begin{array}{ccccc}
\frac{1}{\mathbb{P}_{1}} & \frac{1}{\mathbb{P}_{1}} & \frac{1}{\mathbb{P}_{1}} & \cdots & \frac{1}{\mathbb{P}_{1}} \\
0 & \frac{1}{\mathbb{P}_{2}}-\frac{1}{\mathbb{P}_{1}} & \frac{1}{\mathbb{P}_{2}}-\frac{1}{\mathbb{P}_{1}} & \cdots & \frac{1}{\mathbb{P}_{2}}-\frac{1}{\mathbb{P}_{1}} \\
0 & 0 & \frac{1}{\mathbb{P}_{3}}-\frac{1}{\mathbb{P}_{2}} & \cdots & \frac{1}{\mathbb{P}_{3}}-\frac{1}{\mathbb{P}_{2}} \\
\vdots & \vdots & \vdots & \vdots & \vdots \\
0 & 0 & 0 & \cdots & \frac{1}{\mathbb{P}_{n}}-\frac{1}{\mathbb{P}_{n-1}}
\end{array}\right] .
$$

Thus, we find

$$
\operatorname{det}\left(\mathfrak{P}^{\circ(-1)}\right)=\frac{1}{\mathbb{P}_{1}} \prod_{k=2}^{n}\left(\frac{1}{\mathbb{P}_{k}}-\frac{1}{\mathbb{P}_{k-1}}\right)
$$

So, we get

$$
\operatorname{det}\left(\mathfrak{P}^{\circ(-1)}\right)=\frac{(-1)^{n-1}}{\prod_{i=1}^{n-1} P_{i} \mathbb{P}_{i}^{2} \mathbb{P}_{n}}
$$


Theorem 6. Let $\mathfrak{P}$ be a matrix which is given in (10), then the Euclidean norm of $\mathfrak{P}$ is

$$
\|\mathfrak{P}\|_{\mathbb{E}}=\sqrt{(n+1)^{2} \mathbb{P}_{n+1}^{2}-(2 n+1)+\sum_{k=1}^{n} \frac{(k+1)(k-(2 n+1))}{P_{k+1}}\left(\mathbb{P}_{k}+\mathbb{P}_{k+1}\right)}
$$

Proof. By the Euclidean norm of $\mathfrak{P}$, we get

$$
\|\mathfrak{P}\|_{\mathbb{E}}^{2}=\left[\left(\sum_{i=1}^{n} \sum_{j=1}^{n}\left|\mathbb{P}_{k_{i j}}\right|^{2}\right)^{\frac{1}{2}}\right]^{2}
$$

Thus, we calculate

$$
\|\mathfrak{P}\|_{\mathbb{E}}^{2}=\sum_{k=1}^{n}(2 n-2 k+1) \mathbb{P}_{k}^{2}=(2 n+1) \sum_{k=1}^{n} \mathbb{P}_{k}^{2}-2 \sum_{k=1}^{n} k \mathbb{P}_{k}^{2} .
$$

Furthermore, by the help of the reference [4], we get

$$
\sum_{k=1}^{n} \mathbb{P}_{k}^{2}=\mathbb{P}_{n+1}^{2}(n+1)-1-\sum_{k=1}^{n} \frac{(k+1)}{P_{k+1}}\left(\mathbb{P}_{k}+\mathbb{P}_{k+1}\right),
$$

and

$$
\sum_{k=1}{ }^{n} k \mathbb{P}^{2}{ }_{k}=\mathbb{P}^{2}{ }_{n+1} \frac{n(n+1)}{2}-\sum_{k=1} \frac{(k+1) k}{2 P_{k+1}}\left(\mathbb{P}_{k}+\mathbb{P}_{k+1}\right) .
$$

According to (12) and (13), we obtain

$$
\|\mathfrak{P}\|_{\mathbb{E}}=\sqrt{(n+1)^{2} \mathbb{P}_{n+1}^{2}-(2 n+1)+\sum_{k=1}^{n} \frac{(k+1)(k-(2 n+1))}{P_{k+1}}\left(\mathbb{P}_{k}+\mathbb{P}_{k+1}\right)} .
$$

Corollary 2. Let $\mathfrak{P}$ be a matrix which is given in (10), then the following inequality is obtained:

$$
\begin{gathered}
\frac{1}{\sqrt{n}} \sqrt{(n+1)^{2} \mathbb{P}_{n+1}^{2}-(2 n+1)+\sum_{k=1}^{n} \frac{(k+1)(k-(2 n+1))}{P_{k+1}}\left(\mathbb{P}_{k}+\mathbb{P}_{k+1}\right)} \leq\|\mathfrak{P}\|_{2} \\
\leq \sqrt{(n+1)^{2} \mathbb{P}_{n+1}^{2}-(2 n+1)+\sum_{k=1}^{n} \frac{(k+1)(k-(2 n+1))}{P_{k+1}}\left(\mathbb{P}_{k}+\mathbb{P}_{k+1}\right)} .
\end{gathered}
$$

Proof. The proof can be seen easily by using the proof above and the Inequality (6).

Theorem 7. Let $\mathfrak{P}$ be a matrix as in the matrix form (10). Then, we have the upper bound for the spectral norm of $\mathfrak{P}$ as follows:

$$
\|\mathfrak{P}\|_{2} \leq \sqrt{\left[(n+1) \mathbb{P}_{n+1}^{2}-1-\sum_{k=1}^{n} \frac{(k+1)}{P_{k+1}}\left(\mathbb{P}_{k}+\mathbb{P}_{k+1}\right)\right]\left[n \mathbb{P}_{n}^{2}-\sum_{k=1}^{n-1} \frac{(k+1)}{P_{k+1}}\left(\mathbb{P}_{k}+\mathbb{P}_{k+1}\right)\right]}
$$

Proof. We can write

$$
\mathfrak{P}=\mathbb{A} \circ \mathbb{B},
$$

where

$$
\mathbb{A}=\left[\begin{array}{ccccc}
\mathbb{P}_{1} & 1 & 1 & \cdots & 1 \\
\mathbb{P}_{1} & \mathbb{P}_{2} & 1 & \cdots & 1 \\
\mathbb{P}_{1} & \mathbb{P}_{2} & \mathbb{P}_{3} & \cdots & 1 \\
\vdots & \vdots & \vdots & \vdots & \\
\mathbb{P}_{1} & \mathbb{P}_{2} & \mathbb{P}_{3} & \cdots & \mathbb{P}_{n}
\end{array}\right]
$$


and

$$
\mathbb{B}=\left[\begin{array}{ccccc}
1 & \mathbb{P}_{1} & \mathbb{P}_{1} & \cdots & \mathbb{P}_{1} \\
1 & 1 & \mathbb{P}_{2} & \cdots & \mathbb{P}_{2} \\
1 & 1 & 1 & \cdots & \mathbb{P}_{3} \\
\vdots & \vdots & \vdots & \vdots & \\
1 & 1 & 1 & \cdots & 1
\end{array}\right]
$$

Therefore, we get

$$
\begin{gathered}
\|\mathbb{A}\|_{r_{1}}=\sqrt{\sum_{i=1}^{n} \mathbb{P}_{i}^{2}}=\sqrt{(n+1) \mathbb{P}_{n+1}^{2}-1-\sum_{k=1}^{n} \frac{(k+1)}{P_{k+1}}\left(\mathbb{P}_{k}+\mathbb{P}_{k+1}\right)}, \\
\|\mathbb{B}\|_{c_{1}}=\sqrt{\sum_{i=1}^{n-1} \mathbb{P}_{i}^{2}+1}=\sqrt{n \mathbb{P}_{n}^{2}-\sum_{k=1}^{n-1} \frac{(k+1)}{P_{k+1}}\left(\mathbb{P}_{k}+\mathbb{P}_{k+1}\right) .}
\end{gathered}
$$

Consequently, by using the inequality given in (5), we find

$$
\|\mathfrak{P}\|_{2} \leq \sqrt{\left[(n+1) \mathbb{P}_{n+1}^{2}-1-\sum_{k=1}^{n} \frac{(k+1)}{P_{k+1}}\left(\mathbb{P}_{k}+\mathbb{P}_{k+1}\right)\right]\left[n \mathbb{P}_{n}^{2}-\sum_{k=1}^{n-1} \frac{(k+1)}{P_{k+1}}\left(\mathbb{P}_{k}+\mathbb{P}_{k+1}\right)\right]}
$$

Theorem 8. Assume that $e^{\circ \mathfrak{P}}$ is a matrix as in the form (11), then we calculate

$$
\operatorname{det}\left(e^{\circ \mathfrak{P}}\right)=e^{\mathbb{P}_{1}} \prod_{k=2}^{n}\left(e^{\mathbb{P}_{k}}-e^{\mathbb{P}_{k-1}}\right) .
$$

Proof. The proof can be done easily by applying the elementary row operations.

Theorem 9. Let $e^{\circ \mathfrak{P}}$ be a matrix which is given in (11) and assume that the leading principal minor of $e^{\circ \mathfrak{B}}$ is denoted by $\Delta_{n}$, then we have for $n>1$

i. $\quad \Delta_{n}=\left(e^{\mathbb{P}_{n}}-e^{\mathbb{P}_{n-1}}\right) \Delta_{n-1}$,

ii. $\quad \Delta_{1} \Delta_{2} \Delta_{3} \cdots \Delta_{n}=\left(e^{\mathbb{P}_{1}}\right)^{n}\left(e^{\mathbb{P}_{2}}-e^{\mathbb{P}_{1}}\right)^{n-1}\left(e^{\mathbb{P}_{3}}-e^{\mathbb{P}_{2}}\right)^{n-2} \ldots\left(e^{\mathbb{P}_{n}}-e^{\mathbb{P}_{n-1}}\right)$.

Proof. It can be calculated by using Theorem 8 and the following equations

$$
\begin{gathered}
\Delta_{1}=e, \Delta_{2}=e\left(e^{\frac{3}{2}}-e\right), \Delta_{3}=e\left(e^{\frac{3}{2}}-e\right)\left(e^{\frac{17}{10}}-e^{\frac{3}{2}}\right), \\
\cdots, \Delta_{n}=e^{\mathbb{P}_{1}}\left(e^{\mathbb{P}_{2}}-e^{\mathbb{P}_{1}}\right)\left(e^{\mathbb{P}_{3}}-e^{\mathbb{P}_{2}}\right) \ldots\left(e^{\mathbb{P}_{n}}-e^{\mathbb{P}_{n-1}}\right) .
\end{gathered}
$$

Corollary 3. Let $e^{\circ \mathfrak{P}}$ be a matrix which is given in (11), then $e^{\circ \mathfrak{P}}$ is a positively defined matrix and all eigenvalues of $e^{\circ \mathfrak{P}}$ are positive. 
Theorem 10. Suppose that $e^{\circ \mathfrak{P}}$ is a matrix as in the matrix form (11), then the inverse of the matrix $e^{\circ \mathfrak{P}}$ is denoted by $\left(e^{\circ \mathfrak{P}}\right)^{-1}$ and calculated as

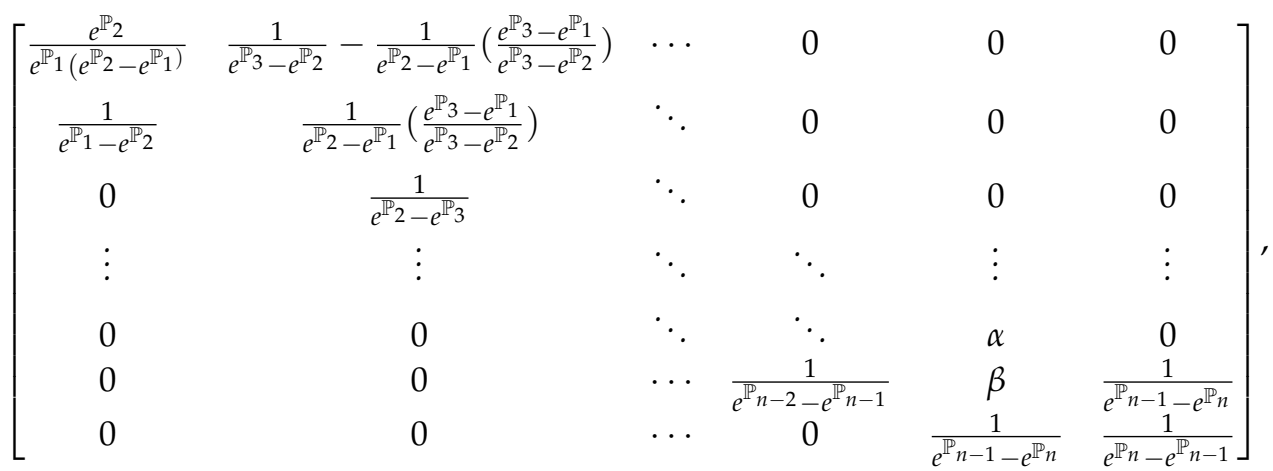

where

$$
\alpha=\frac{1}{e^{\mathbb{P}_{n}}-e^{\mathbb{P}_{n-1}}}-\frac{1}{e^{\mathbb{P}_{n-1}}-e^{\mathbb{P}_{n-2}}}\left(\frac{e^{\mathbb{P}_{n}}-e^{\mathbb{P}_{n-2}}}{e^{\mathbb{P}_{n}}-e^{\mathbb{P}_{n-1}}}\right),
$$

and

$$
\beta=\frac{1}{e^{\mathbb{P}_{n-1}-e^{\mathbb{P}_{n-2}}}}\left(\frac{e^{\mathbb{P}_{n}}-e^{\mathbb{P}_{n-2}}}{e^{\mathbb{P}_{n}}-e^{\mathbb{P}_{n-1}}}\right)
$$

Proof. By following the same way in Theorem 3, the proof can be seen easily.

Theorem 11. Assume that $e^{\mathfrak{P}}$ is a matrix which is given in (11). Then,

$$
\operatorname{det}\left(e^{\mathfrak{P} \circ(-1)}\right)=\frac{1}{e^{\mathbb{P}_{1}}} \prod_{k=2}^{n} \frac{1}{e^{\mathbb{P}_{k-1}}} \frac{\left(1-e^{\frac{1}{P_{k}}}\right)}{e^{\frac{1}{P_{k}}}}
$$

Proof. By the definition of the Hadamard inverse matrix, we define

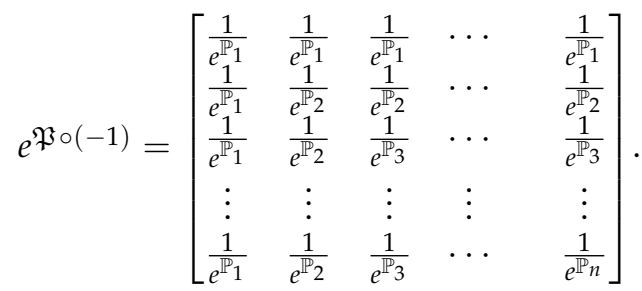

By the elementary row operations, we get

$$
\operatorname{det}\left(e^{\mathfrak{P} \circ(-1)}\right)=\frac{1}{e^{\mathbb{P}_{1}}}\left(\prod_{k=2}\right)^{n}\left(\frac{1}{e^{\mathbb{P}} k}-\frac{1}{e^{\mathbb{P}_{k-1}}}\right)=\frac{1}{e^{\mathbb{P}_{1}}} \prod_{k=2}{ }^{n} \frac{1}{e^{\mathbb{P}_{k-1}}} \frac{\left(1-e^{\frac{1}{P_{k}}}\right.}{e^{\frac{1}{P_{k}}}} .
$$

Theorem 12. Let us consider the matrix $e^{\circ \mathfrak{P}}$ given by (11), then

$$
\left\|e^{\circ \mathfrak{P}}\right\|_{\mathbb{E}}=\sqrt{(2 n+1) \sum_{k=1}^{n} e^{2 \mathbb{P}_{k}}-2 \sum_{k=1}^{n} k e^{2 \mathbb{P}_{k}}}
$$

Proof. By exploiting the definition of Euclidean norm,

$$
\left\|e^{\circ \mathfrak{P}}\right\|_{\mathbb{E}}^{2}=\sum_{k=1}^{n}(2 n-2 k+1) e^{2 \mathbb{P}_{k}}=(2 n+1) \sum_{k=1}^{n} e^{2 \mathbb{P}_{k}}-2 \sum_{k=1}^{n} k e^{2 \mathbb{P}_{k}} .
$$

So, the proof is clear. 
Corollary 4. Let $e^{\circ \mathfrak{P}}$ be a matrix as in the matrix form (11). Then, the following inequalities are hold:

$$
\frac{1}{\sqrt{n}} \sqrt{(2 n+1) \sum_{k=1}^{n} e^{2 \mathbb{P}_{k}}-2 \sum_{k=1}^{n} k e^{2 \mathbb{P}_{k}}} \leq\left\|e^{\circ \mathfrak{P}}\right\|_{2} \leq \sqrt{(2 n+1) \sum_{k=1}^{n} e^{2 \mathbb{P}_{k}}-2 \sum_{k=1}^{n} k e^{2 \mathbb{P}_{k}}}
$$

Proof. The proof can be seen easily by using Theorem 12 and the Inequality (6).

Theorem 13. Assume that $e^{\circ \mathfrak{P}}$ is a matrix as in the matrix form (11). Then, we have an upper bound for the spectral norm of the matrix $e^{\circ \mathfrak{P}}$ as follow:

$$
\left\|e^{\circ \mathfrak{P}}\right\|_{2} \leq \sqrt{\left(\frac{e^{2 \mathbb{P}_{n+1}}-e^{2}}{2(e-1)}\right)\left(\frac{e^{2 \mathbb{P}_{n}}-e^{2}}{2(e-1)}+1\right)} .
$$

Proof. We can write

$$
e^{\circ \mathfrak{P}}=\mathfrak{A} \circ \mathfrak{B}
$$

where

$$
\mathfrak{A}=\left[\begin{array}{ccccc}
e^{\mathbb{P}_{1}} & 1 & 1 & \cdots & 1 \\
e^{\mathbb{P}_{1}} & e^{\mathbb{P}_{2}} & 1 & \cdots & 1 \\
e^{\mathbb{P}_{1}} & e^{\mathbb{P}_{2}} & e^{\mathbb{P}_{3}} & \cdots & 1 \\
\vdots & \vdots & \vdots & \vdots & \vdots \\
e^{\mathbb{P}_{1}} & e^{\mathbb{P}_{2}} & e^{\mathbb{P}_{3}} & \cdots & e^{\mathbb{P}_{n}}
\end{array}\right]
$$

and

$$
\mathfrak{B}=\left[\begin{array}{ccccc}
1 & e^{\mathbb{P}_{1}} & e^{\mathbb{P}_{1}} & \cdots & e^{\mathbb{P}_{1}} \\
1 & 1 & e^{\mathbb{P}_{2}} & \cdots & e^{\mathbb{P}_{2}} \\
1 & 1 & 1 & \cdots & e^{\mathbb{P}_{3}} \\
\vdots & \vdots & \vdots & \vdots & \vdots \\
1 & 1 & 1 & \cdots & 1
\end{array}\right]
$$

So,

$$
\|\mathfrak{A}\|_{r_{1}}=\sqrt{\sum_{k=1}^{n} e^{2 \mathbb{P}_{k}}}
$$

and

$$
\|\mathfrak{B}\|_{c_{1}}=\sqrt{\sum_{k=1}^{n-1} e^{2 \mathbb{P}_{k}}+1}
$$

Thus, according to (5) we obtain

$$
\left\|e^{\circ \mathfrak{P}}\right\|_{2} \leq \sqrt{\left(\sum_{k=1}^{n} e^{2 \mathbb{P}_{k}}\right)\left(\sum_{k=1}^{n-1} e^{2 \mathbb{P}_{k}}+1\right)}
$$

\section{Numerical Examples}

In this section, we present an illustrative example that we calculate all results for the symmetric $5 \times 5$ matrix whose entries are the harmonic Pell numbers. 
Example 1. Let $\mathfrak{P}$ be a matrix as in matrix form (10) for $n=5$. Then, the matrix $\mathfrak{P}$ is

$$
\mathfrak{P}=\left[\begin{array}{ccccc}
1 & 1 & 1 & 1 & 1 \\
1 & \frac{3}{2} & \frac{3}{2} & \frac{3}{2} & \frac{3}{2} \\
1 & \frac{3}{2} & \frac{17}{10} & \frac{17}{10} & \frac{17}{10} \\
1 & \frac{3}{2} & \frac{17}{10} & \frac{107}{60} & \frac{107}{60} \\
1 & \frac{3}{2} & \frac{17}{10} & \frac{107}{60} & \frac{449}{247}
\end{array}\right]
$$

which can be found from the MATLAB code in Table 2. From the Theorem 1, the determinant of $\mathfrak{P}$ can be calculated as

$$
\operatorname{det}(\mathfrak{P})=\frac{1}{P_{1} P_{2} P_{3} P_{4} P_{5}}=\frac{1}{3480} .
$$

The inverse of the matrix $\mathfrak{P}$ and the Hadamard inverse of $\mathfrak{P}$ can be written as

$$
\mathfrak{P}^{-1}=\left[\begin{array}{ccccc}
P_{1}+P_{2} & -P_{2} & 0 & 0 & 0 \\
-P_{2} & P_{2}+P_{3} & -P_{3} & 0 & 0 \\
0 & -P_{3} & P_{3}+P_{4} & -P_{4} & 0 \\
0 & 0 & -P_{4} & P_{4}+P_{5} & -P_{5} \\
0 & 0 & 0 & -P_{5} & P_{5}
\end{array}\right]=\left[\begin{array}{ccccc}
3 & -2 & 0 & 0 & 0 \\
-2 & 7 & -5 & 0 & 0 \\
0 & -5 & 17 & -12 & 0 \\
0 & 0 & -12 & 41 & -29 \\
0 & 0 & 0 & -29 & 29
\end{array}\right]
$$

and

$$
\mathfrak{P}^{\circ(-1)}=\left[\begin{array}{lllll}
\frac{1}{\mathbb{P}_{1}} & \frac{1}{\mathbb{P}_{1}} & \frac{1}{\mathbb{P}_{1}} & \frac{1}{\mathbb{P}_{1}} & \frac{1}{\mathbb{P}_{1}} \\
\frac{1}{\mathbb{P}_{1}} & \frac{1}{\mathbb{P}_{2}} & \frac{1}{\mathbb{P}_{2}} & \frac{1}{\mathbb{P}_{2}} & \frac{1}{\mathbb{P}_{2}} \\
\frac{1}{\mathbb{P}_{1}} & \frac{1}{\mathbb{P}_{2}} & \frac{1}{\mathbb{P}_{3}} & \frac{1}{\mathbb{P}_{3}} & \frac{1}{\mathbb{P}_{3}} \\
\frac{1}{\mathbb{P}_{1}} & \frac{1}{\mathbb{P}_{2}} & \frac{1}{\mathbb{P}_{3}} & \frac{1}{\mathbb{P}_{4}} & \frac{1}{\mathbb{P}_{4}} \\
\frac{1}{\mathbb{P}_{1}} & \frac{1}{\mathbb{P}_{2}} & \frac{1}{\mathbb{P}_{3}} & \frac{1}{\mathbb{P}_{4}} & \frac{1}{\mathbb{P}_{5}}
\end{array}\right]=\left[\begin{array}{ccccc}
1 & 1 & 1 & 1 & 1 \\
1 & \frac{2}{3} & \frac{2}{3} & \frac{2}{3} & \frac{2}{3} \\
1 & \frac{2}{3} & \frac{10}{17} & \frac{10}{17} & \frac{10}{17} \\
1 & \frac{2}{3} & \frac{10}{17} & \frac{60}{107} & \frac{60}{107} \\
1 & \frac{2}{3} & \frac{10}{17} & \frac{60}{107} & \frac{247}{449}
\end{array}\right],
$$

respectively. By exploiting the contraction method from Theorem 4, we obtain the permanent of the matrix $\mathfrak{P}^{-1}$ as follows:

$$
\left[\mathfrak{P}^{-1}\right]^{(1)}=\left[\begin{array}{cccc}
A & -P_{3}\left(P_{1}+P_{2}\right) & 0 & 0 \\
-P_{3} & P_{3}+P_{4} & -P_{4} & 0 \\
0 & -P_{4} & P_{4}+P_{5} & -P_{5} \\
0 & 0 & -P_{5} & P_{5}
\end{array}\right]=\left[\begin{array}{cccc}
25 & -15 & 0 & 0 \\
-5 & 17 & -12 & 0 \\
0 & -12 & 41 & -29 \\
0 & 0 & -29 & 29
\end{array}\right] .
$$

Going on with the method:

$$
\left[\mathfrak{P}^{-1}\right]^{(2)}=\left[\begin{array}{ccc}
v^{(0)} & -A P_{4} & 0 \\
-P_{4} & P_{4}+P_{5} & -P_{5} \\
0 & -P_{5} & P_{5}
\end{array}\right]=\left[\begin{array}{ccc}
500 & -6000 & 0 \\
-12 & 41 & -29 \\
0 & -29 & 29
\end{array}\right],
$$

where $v^{(0)}=P_{3}^{2}\left(P_{1}+P_{2}\right)+A\left(P_{3}+P_{4}\right)$. Finally, we get:

$$
\left[\mathfrak{P}^{-1}\right]^{(3)}=\left[\begin{array}{cc}
v^{(1)} & -P_{5} \mathfrak{v}^{(0)} \\
-P_{5} & P_{5}
\end{array}\right]=\left[\begin{array}{cc}
24100 & -14500 \\
-29 & 29
\end{array}\right]
$$

where $v^{(1)}=A P_{4}^{2}+v^{(0)}\left(P_{4}+P_{5}\right)$. So, the permanent of the matrix $\mathfrak{P}^{-1}$ is

$$
\operatorname{per}\left(\mathfrak{P}^{-1}\right)=P_{5}\left(v^{(1)}+P_{5} v^{(0)}\right)=1119400 .
$$

From Theorem 5 , the determinant of $\mathfrak{P}^{\circ(-1)}$ is

$$
\operatorname{det}\left(\mathfrak{P}^{\circ(-1)}\right)=\frac{80000}{10465611043} \approx 7.644 \times 10^{-6} .
$$


By the light of Theorem 6, one can calculate the Euclidean norm of the matrix $\mathfrak{P}$ as

$$
\|\mathfrak{P}\|_{E} \approx 7.214 \text {. }
$$

For the spectral norm of $\mathfrak{P}$, we can write the following inequality from Corollary 2:

$$
3.226 \leq\|\mathfrak{P}\|_{2} \leq 7.214 \text {. }
$$

Moreover, an upper bound for the spectral norm can be found from Theorem 7 as follows:

$$
\|\mathfrak{P}\|_{2} \leq \sqrt{\frac{11205}{86}} \approx 11.414 .
$$

For finding this bound, we use two auxiliary matrices via Theorem 7 . These matrices are

$$
\mathbb{A}=\left[\begin{array}{ccccc}
\mathbb{P}_{1} & 1 & 1 & 1 & 1 \\
\mathbb{P}_{1} & \mathbb{P}_{2} & 1 & 1 & 1 \\
\mathbb{P}_{1} & \mathbb{P}_{2} & \mathbb{P}_{3} & 1 & 1 \\
\mathbb{P}_{1} & \mathbb{P}_{2} & \mathbb{P}_{3} & \mathbb{P}_{4} & 1 \\
\mathbb{P}_{1} & \mathbb{P}_{2} & \mathbb{P}_{3} & \mathbb{P}_{4} & \mathbb{P}_{5}
\end{array}\right]=\left[\begin{array}{ccccc}
1 & 1 & 1 & 1 & 1 \\
1 & \frac{3}{2} & 1 & 1 & 1 \\
1 & \frac{3}{2} & \frac{17}{10} & 1 & 1 \\
1 & \frac{3}{2} & \frac{17}{10} & \frac{107}{60} & 1 \\
1 & \frac{3}{2} & \frac{17}{10} & \frac{107}{60} & \frac{449}{247}
\end{array}\right]
$$

and

$$
\mathbb{B}=\left[\begin{array}{ccccc}
1 & \mathbb{P}_{1} & \mathbb{P}_{1} & \mathbb{P}_{1} & \mathbb{P}_{1} \\
1 & 1 & \mathbb{P}_{2} & \mathbb{P}_{2} & \mathbb{P}_{2} \\
1 & 1 & 1 & \mathbb{P}_{3} & \mathbb{P}_{3} \\
1 & 1 & 1 & 1 & \mathbb{P}_{4} \\
1 & 1 & 1 & 1 & 1
\end{array}\right]=\left[\begin{array}{ccccc}
1 & 1 & 1 & 1 & 1 \\
1 & 1 & \frac{3}{2} & \frac{3}{2} & \frac{3}{2} \\
1 & 1 & 1 & \frac{17}{10} & \frac{17}{10} \\
1 & 1 & 1 & 1 & \frac{107}{60} \\
1 & 1 & 1 & 1 & 1
\end{array}\right]
$$

The maximum row norm of the matrix $\mathbb{A}$ is

$$
\|\mathbb{A}\|_{r_{1}}=\sqrt{\frac{2509239697}{219632400}} \approx 3.38
$$

and the maximum column norm of the matrix $\mathbb{B}$ is

$$
\|\mathbb{B}\|_{c_{1}}=\sqrt{\frac{40033}{3600}} \approx 3.33 .
$$

By using the definition of it, the Hadamard exponential matrix of $\mathfrak{P}$ is constructed as

$$
e^{\circ \mathfrak{P}}=\left[\begin{array}{ccccc}
e & e & e & e & e \\
e & e^{\frac{3}{2}} & e^{\frac{3}{2}} & e^{\frac{3}{2}} & e^{\frac{3}{2}} \\
e & e^{\frac{3}{2}} & e^{\frac{17}{10}} & e^{\frac{17}{10}} & e^{\frac{17}{10}} \\
e & e^{\frac{3}{2}} & e^{\frac{17}{10}} & e^{\frac{107}{60}} & e^{\frac{107}{60}} \\
e & e^{\frac{3}{2}} & e^{\frac{17}{10}} & e^{\frac{107}{60}} & e^{\frac{499}{247}}
\end{array}\right] .
$$

So, the determinant of $e^{\circ \mathfrak{P}}$ can be calculated from Theorem 8 as

$$
\operatorname{det}\left(e^{\circ \mathfrak{P}}\right)=\frac{613}{1298} \approx 0.472 .
$$

The inverse of the matrix $e^{\circ \mathfrak{P}}$ and the Hadamard inverse of $e^{\circ \mathfrak{P}}$ can be written as

$$
\left(e^{\circ \mathfrak{P}}\right)^{-1}=\left[\begin{array}{ccccc}
\frac{115}{123} & -\frac{93}{164} & 0 & 0 & 0 \\
-\frac{93}{164} & \frac{326}{207} & -\frac{129}{128} & 0 & 0 \\
0 & -\frac{129}{128} & \frac{877}{282} & -\frac{494}{235} & 0 \\
0 & 0 & -\frac{494}{235} & \frac{4694}{681} & -\frac{206}{43} \\
0 & 0 & 0 & -\frac{206}{43} & \frac{206}{43}
\end{array}\right]
$$


and

$$
e^{\mathfrak{P} \circ(-1)}=\left[\begin{array}{ccccc}
e & e & e & e & e \\
e & e^{-\frac{3}{2}} & e^{-\frac{3}{2}} & e^{-\frac{3}{2}} & e^{-\frac{3}{2}} \\
e & e^{-\frac{3}{2}} & e^{-\frac{17}{10}} & e^{-\frac{17}{10}} & e^{-\frac{17}{10}} \\
e & e^{-\frac{3}{2}} & e^{-\frac{17}{10}} & e^{-\frac{107}{60}} & e^{-\frac{107}{60}} \\
e & e^{-\frac{3}{2}} & e^{-\frac{17}{10}} & e^{-\frac{107}{60}} & e^{-\frac{449}{247}}
\end{array}\right],
$$

respectively. From Theorem 11, the determinant of $e^{\mathfrak{P} \circ(-1)}$ can be stated as follows:

$$
\operatorname{det}\left(e^{\mathfrak{P} \circ(r-1)}\right) \approx 1.792 \times 10^{-7} .
$$

By the light of Theorem 12 or by using the MATLAB code in Table 2, one can calculate the Euclidean norm of the matrix $e^{\circ \mathfrak{P}}$ as

$$
\left\|e^{\circ \mathfrak{P}}\right\|_{\mathbb{E}} \approx 22.384 .
$$

For the spectral norm of $e^{\circ \mathfrak{P}}$, we can write the following inequality from Corollary 4:

$$
10.011 \leq\left\|e^{\circ \mathfrak{P}}\right\|_{2} \leq 22.384 .
$$

Moreover, an upper bound for the spectral norm can be found from Theorem 13 or the MATLAB code in Table 2 as follows:

$$
\left\|e^{\circ \mathfrak{P}}\right\|_{2} \leq 110.772 .
$$

For finding this bound, we use two auxiliary matrices via Theorem 13. These matrices are

$$
\mathfrak{A}=\left[\begin{array}{ccccc}
e^{\mathbb{P}_{1}} & 1 & 1 & 1 & 1 \\
e^{\mathbb{P}_{1}} & e^{\mathbb{P}_{2}} & 1 & 1 & 1 \\
e^{\mathbb{P}_{1}} & e^{\mathbb{P}_{2}} & e^{\mathbb{P}_{3}} & 1 & 1 \\
e^{\mathbb{P}_{1}} & e^{\mathbb{P}_{2}} & e^{\mathbb{P}_{3}} & e^{\mathbb{P}_{4}} & 1 \\
e^{\mathbb{P}_{1}} & e^{\mathbb{P}_{2}} & e^{\mathbb{P}_{3}} & e^{\mathbb{P}_{4}} & e^{\mathbb{P}_{5}}
\end{array}\right]=\left[\begin{array}{ccccc}
e & 1 & 1 & 1 & 1 \\
e & e^{\frac{3}{2}} & 1 & 1 & 1 \\
e & e^{\frac{3}{2}} & e^{\frac{17}{10}} & 1 & 1 \\
e & e^{\frac{3}{2}} & e^{\frac{17}{10}} & e^{\frac{107}{60}} & 1 \\
e & e^{\frac{3}{2}} & e^{\frac{17}{10}} & e^{\frac{107}{60}} & e^{\frac{449}{247}}
\end{array}\right]
$$

and

$$
\mathfrak{B}=\left[\begin{array}{ccccc}
1 & e^{\mathbb{P}_{1}} & e^{\mathbb{P}_{1}} & e^{\mathbb{P}_{1}} & e^{\mathbb{P}_{1}} \\
1 & 1 & e^{\mathbb{P}_{2}} & e^{\mathbb{P}_{2}} & e^{\mathbb{P}_{2}} \\
1 & 1 & 1 & e^{\mathbb{P}_{3}} & e^{\mathbb{P}_{3}} \\
1 & 1 & 1 & 1 & e^{\mathbb{P}_{4}} \\
1 & 1 & 1 & 1 & 1
\end{array}\right]=\left[\begin{array}{ccccc}
1 & e & e & e & e \\
1 & 1 & e^{\frac{3}{2}} & e^{\frac{3}{2}} & e^{\frac{3}{2}} \\
1 & 1 & 1 & e^{\frac{17}{10}} & e^{\frac{17}{10}} \\
1 & 1 & 1 & 1 & e^{\frac{107}{60}} \\
1 & 1 & 1 & 1 & 1
\end{array}\right] .
$$

From Theorem 13 or the following MATLAB code, the maximum row norm of the matrix $\mathfrak{A}$ can be calculated as

$$
\|\mathfrak{A}\|_{r_{1}} \approx 130.763
$$

and the maximum column norm of the matrix $\mathfrak{B}$ can be found as

$$
\|\mathfrak{B}\|_{c_{1}} \approx 93.837 \text {. }
$$


Table 2. MATLAB-R2016a code for the matrix $\mathfrak{P}$ and for the norm calculations of $e^{\circ \mathfrak{P}}$.

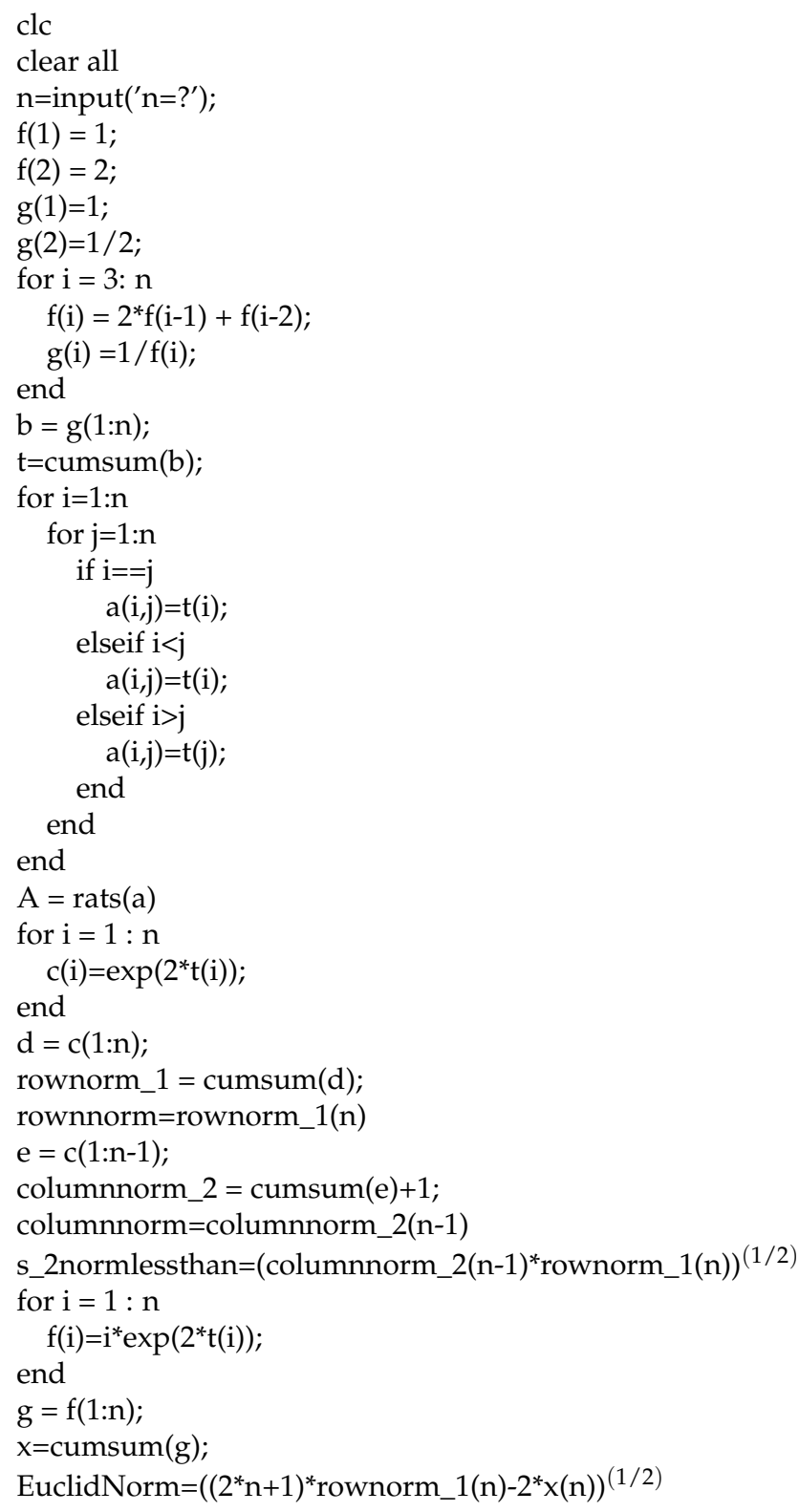

\section{Conclusions}

In this paper, we define a new number sequence named harmonic Pell numbers. Then, we construct a special symmetric matrix $\mathfrak{P}$ whose entries are the harmonic Pell numbers and its Hadamard exponential matrix $e^{\circ \mathfrak{P}}$. We examine some linear algebraic properties of the matrices and state some bounds for the Euclidean and spectral norms of them. Furthermore, we obtain some summation formulas for the harmonic Pell numbers, which we think can shed light on the studies that can be done about the harmonic Pell numbers in the future. Moreover, we give a MATLAB-R2016a code for the matrix $\mathfrak{P}$ and for the norm calculations of $e^{\circ \mathfrak{P}}$ (See, Table 2). Here, we prefer writing a new algorithm at MATLAB-R2016a code which is not included in the standard Matlab libraries. For the value $n$ inputted to the code given

1. writes the matrix $\mathfrak{P}$,

2. for the matrix $e^{\circ \mathfrak{P}}$,

i. calculates the row norm $\|\mathfrak{A}\|_{r_{1}}$,

ii. calculates the column norm $\|\mathfrak{B}\|_{c_{1}}$, 
iii. gives an upper bound for the spectral norm $\left\|e^{\circ \mathfrak{P}}\right\|_{2}$,

iv. obtains $\left\|e^{\circ \mathfrak{P}}\right\|_{\mathbb{E}}$.

Author Contributions: Conceptualization, S.Y.A., M.A. and F.Y.; methodology, S.Y.A. and M.A.; software, M.A.; validation, S.Y.A.; formal analysis, S.Y.A. and M.A.; investigation, S.Y.A., M.A. and F.Y.; resources, M.A. and F.Y.; data curation, S.Y.A., M.A. and F.Y.; writing-original draft preparation, S.Y.A.; writing-review and editing, S.Y.A., M.A. and F.Y.; visualization, M.A.; supervision, S.Y.A., M.A. and F.Y.; project administration, S.Y.A. All authors have read and agreed to the published version of the manuscript.

Funding: This research received no external funding.

Acknowledgments: The authors appreciate anonymous referees for their careful corrections to and valuable comments on the paper.

Conflicts of Interest: The authors declare no conflict of interest.

\section{References}

1. Horadam, A.F. Pell identities. Fibonacci Q. 1971, 9, 245-252.

2. Radičić, B. On k-Circulant Matrices Involving the Pell Numbers. Results Math. 2019, 74, 200. [CrossRef]

3. Andrade, E.; Carrasco-Olivera, D.; Manzaneda, C. On circulant like matrices properties involving Horadam, Fibonacci, Jacobsthal and Pell numbers. Linear Algebra Appl. 2021, 617, 100-120. [CrossRef]

4. Graham, R.; Knuth, D.; Patashnik, K. Concrete Mathematics; Addisson-Wesley: Reading, UK, 1989.

5. Bahsi, M.; Solak, S. On the matrices with Harmonic numbers. GU. J. Sc. 2010, 23, 445-448.

6. Das, K.C.; da Fonseca, C.M. Some spectral bounds for the harmonic matrix. Şt. Univ. Ovidius Constanţa 2017, 25, 73-81. [CrossRef]

7. Tuglu, N.; Kızılateş, C. On the norms of circulant and r-circulant matrices with the hyperharmonic Fibonacci numbers. J. Inequal. Appl. 2015, 2015, 253. [CrossRef]

8. Zhang, F. Matrix Theory; Basic Results and Techniques; Springer: New York, NY, USA, 1999.

9. Horn, RA.; Johnson, C.R. Matrix Analysis, Cambridge [Cambridge Shire]; Cambridge University Press: New York, NY, USA, 1985.

10. Reams, R. Hadamard inverses, square roots and products of almost semidefinite matrices. Linear Algebra Appl. 1999, 288 , 35-43. [CrossRef]

11. Akbulak, M.; Ipek, A. Hadamard exponential Hankel Matrix, its eigenvalues and some norms. Math. Sci. Lett. $2012,1,81-87$.

12. Bozkurt, D. A note on the spectral norms of the matrices connected integer numbers sequence. Math. GM 2011, 1724, 171-190.

13. Solak, S.; Bahsi, M. On the spectral norms of Toeplitz matrices with Fibonacci and Lucas numbers. Nonlinear Anal. Hacet. J. Math. Stat. 2013, 42, 15-19.

14. Marvin, M.; Henryk, M. Permanents. Am. Math. Mon. 1965, 72, 577-591. [CrossRef]

15. Brualdi, R.A.; Gibson, P.M. Convex polyhedra of doubly stochastic matrices I: Applications of the permanent function. J. Combin. Theory A 1977, 22, 194-230. [CrossRef]

16. Lehmer, D.H. Fibonacci and related sequences in periodic tridiagonal matrices. Fibonacci Q. 1975, 12, 150-158.

17. Yilmaz, F.; Bozkurt, D. Hessenberg matrices and the Pell and Perrin numbers. J. Number Theory 2011, 131, 1390-1396. [CrossRef]

18. Petroudi, S.H.J.; Pirouz, B. A particular matrix, Its inversion and some norms. Appl. Comput. Math. 2015, 4, 47-52. [CrossRef] 